edu11\&titulo $=$ Alumnado $\% 2520$ con $\% 2520$ trastorno $\% 25$ 20cr\%25F3nico.\%2520Elementos\% 2520para\%2520una\% 2520intervenci $\%$ 25F3n $\% 2520$ psicoeducativa.

12. Salerno M, Militerni R, Di MaioS, et al. Intellectual outcome at 12 years of age in congenital hypothyroidism. Eur J Endocrinol 1999;141:105-10.

13. Sastre-Riba S. Condiciones temprana del desarrollo y el aprendizaje: el papel de las funciones ejecutivas. Rev Neurol
2006;42(Supl 2):S143-51.

14. Álvarez-González MA, Caravajal-MartínezF, Pérez-Gesén $\mathrm{C}$, et al. Pronóstico de la cognición en el hipotiroidismo congénito tratado precozmente. Hipótesis del doble efecto. Rev Neurol 2004;38(6):513-7.

15. Boekaerts M, Röder I. Stress, Coping, and adjustment in children with a chronic disease: a review of the literature. Disabil Rehabil 1999;21(7):311-37.

\title{
Contenido de vitamina A en la leche materna madura y su adecuación a las recomendaciones nutricionales en el lactante
} Vitamin A content in mature breast milk and its adequacy to the nutritional recommendations for infants

\author{
Bioq. Natalia Matamoros ${ }^{a}$, Bioq. Silvana Visentin ${ }^{a}$, Lic. Guillermina Ferrari ${ }^{a}$, Dra. Mariana Falivene, \\ Dra. Victoria Fasano ${ }^{a, b}$ y Dr. Horacio F. González ${ }^{a}$
}

\section{RESUMEN}

Objetivo. Determinar el contenido de vitamina A en la leche materna y evaluar si cubre las recomendaciones para lactantes. Material y métodos. Estudio observacional, prospectivo, de corte transversal. Se analizaron muestras de leche de madres asistidas en el hospital público, obtenidas entre los 30 y los 90 días posparto. Se determinó la concentración de vitamina A por cromatografía y su adecuación a la ingesta dietética recomendada.Seutilizó el coeficiente deSpearman para estudiar la correlación entre variables.

Resultados. Participaron 79 madres. La concentración de vitamina A en las leches fue 1,80 $\mu \mathrm{mol} / \mathrm{L}(1,36-2,30)$; el tiempo medio de lactancia, de 57 días. No se encontró correlación significativa entre días de lactancia y contenido de vitamina A.El contenidoen $50 \%$ delas muestras no cubriólas recomendaciones de vitamina A para los lactantes.

Conclusión. El contenido de vitamina A fue insuficiente para cubrir las recomendaciones en la mitad de los casos.

Palabras clave: vitamina A, lactancia materna, leche humana.

a. Instituto de Desarrollo e Investigaciones Pediátricas "Prof. Dr. Fernando E. Viteri" (IDIP), Hospital de Niños "Sor María Ludovica" de La Plata, Ministerio de Salud/ Comisión de Investigaciones Científicas de la Provincia de Buenos Aires.

b. Departamento de Matemática, Facultad de Ciencias Exactas, Universidad Nacional de La Plata (UNLP).

\section{Correspondencia:}

Dr. Horacio González: horaciofgonzalez@gmail.com

Financiamiento: El estudio fue financiado con recursos de la Institución (Instituto de Desarrollo e Investigaciones Pediátricas "Prof. Dr. Fernando E. Viteri”, IDIP).

Conflicto de intereses: Ninguno que declarar.

Recibido: 26-5-2017

Aceptado: 12-9-2017 http: / / dx.doi.org/10.5546/ aap.2018.146

Texto completo en inglés:

http:/ / dx.doi.org/10.5546/ aap.2018.eng.146

Cómo citar: Matamoros N, Visentin S, Ferrari G, et al. Contenido de vitamina $\mathrm{A}$ en la leche materna madura y su adecuación a las recomendaciones nutricionales en el lactante. Arch Argent Pediatr 2018;116(2):146-149.

\section{INTRODUCCIÓN}

El término "vitamina A" incluye el retinol (vitamina A preformada) y los carotenoides provitamina $\mathrm{A}$, que son los precursores dietarios del retinol. La vitamina ingerida se almacena, principalmente, en el hígado. Cuando se hace necesaria, el retinol hepático se moviliza unido a una proteína transportadora de retinol a través de la sangre para cubrir las necesidades fisiológicas del cuerpo y, en las madres en período de lactancia, incorporarse a la leche materna. ${ }^{1,2}$ El contenido de vitamina A en la leche materna depende del estado nutricional de la madre. ${ }^{3}$

El feto, el neonato y el lactante deben recibir cantidades suficientes de vitamina A para sostener el óptimo desarrollo visual, inmunológico y cognitivo. ${ }^{4}$

Los lactantes nacen con escasos depósitos de vitamina A en el hígado. Esto es debido al control homeostático materno, que regula la transferencia placentaria de vitamina A al feto para evitar que altas concentraciones de esta sean transferidas y resulten nocivas para el niño. Por lo tanto, la leche 
materna es la única fuente de vitamina A desde el nacimiento hasta los 6 meses de edad, ya sea para satisfacer sus necesidades como para construir sus reservas. ${ }^{5}$

El objetivo de este trabajo fue determinar la concentración de vitamina A en la leche materna madura y evaluar su adecuación a las recomendaciones establecidas para el lactante.

\section{PACIENTES Y METODOLOGÍA}

Diseño del estudio y protocolo: Estudio observacional, analítico, prospectivo, de corte transversal.

Criterios de inclusión: Fueron invitadas a participar del estudio las madres que realizaron sus consultas y controles de salud en el observatorio del Instituto de Desarrollo e Investigaciones Pediátricas "Prof. Dr. Fernando E. Viteri" (IDIP), durante el período 2012-2013, desde el primer mes posparto. Las muestras de leche fueron obtenidas de madres, mayores de 18 años, con lactancia materna exclusiva, entre los 30 y los 90 días posparto, con antecedentes fetonatales normales y sin tratamiento vitamínico. Los datos para caracterizar a la población de estudio se obtuvieron de las historias clínicas.

El protocolo fue aprobado por el Comité Institucional de Revisión de Protocolos de Investigación del IDIP y se les hizo firmar un consentimiento informado a las madres que aceptaron participar del estudio. Se llevó a cabo en conformidad con las normas éticas establecidas en la Declaración Universal de Derechos Humanos de 1948, el Código de Núremberg y la Declaración de Helsinki de 1964 y sucesivas revisiones y enmiendas.

\section{Obtención, transporte y conservación de las muestras de leche materna}

La recolección de la leche materna se realizó entre las 9:00 y las 12:00 a. m. con una bomba eléctrica para extracción de leche del seno materno (Medela) hasta el vaciado completo de una mama. Las muestras se conservaron en un freezer a $-70{ }^{\circ} \mathrm{C}$, protegidas de la luz con papel de aluminio hasta su procesamiento.

\section{Determinación del contenido de vitamina $\mathrm{A}$ en la leche materna}

Para determinar el contenido de vitamina A en la leche, se utilizó el procedimiento recomendado para la determinación de retinol total en tejidos de la Sociedad Española de Bioquímica Clínica y Patología Molecular (SEQC). ${ }^{6}$
Las muestras se analizaron por cromatografía líquida de alta performance ultra rápida (ultrafast liquid chromatography; UFLC, por sus siglas en inglés), con detector de arreglo de diodos, longitud de onda de absorción máxima, $\lambda$ máx. $=325 \mathrm{~nm}$. La identificación y cuantificación de retinol en las muestras se estableció por comparación con los tiempos de retención y áreas de un estándar de all-trans-retinol-SIGMA.

Los datos se expresaron en $\mu \mathrm{mol} / \mathrm{L}$.

\section{Tamaño muestral}

El tamaño muestral fue calculado para estimar la concentración media de vitamina A en la leche madura con una confianza del $95 \%$ y un error de $0,10 \mu \mathrm{mol} / \mathrm{L}$, considerando un desvío de $0,47 \mu \mathrm{mol} / \mathrm{L} .{ }^{7}$

El tamaño final estimado fue de 78 muestras de leche.

\section{Análisis de los resultados}

Se analizó la adecuación a las recomendaciones nutricionales en los lactantes utilizando las ingestas diarias recomendadas (dietary reference intakes; DRI, por sus siglas en inglés) para niños nacidos a término y de 0 a 6 meses de edad, dada por el Instituto de Medicina de la Academia Nacional de Ciencias de los Estados Unidos. ${ }^{8}$

El análisis estadístico se realizó mediante el Programa Estadístico SPSS 18 para Windows. Las variables cuantitativas se expresaron como mediana con rango intercuartil (RIQ) y se utilizó el coeficiente de Spearman para estudiar la correlación entre variables.

\section{RESULTADOS}

Se estudiaron 79 muestras de leche madura, donadas por madres [mediana de edad de 25 años (RIQ: 20-30)], con lactancia materna exclusiva. Del relevamiento de las historias clínicas de las madres, se obtuvieron los siguientes resultados: $62,0 \%$ tenían un índice de masa muscular (IMC) superior a 25 y $92,4 \%$ de ellas presentaron un porcentaje de grasa corporal mayor de 30 . Ninguna de las madres presentaba bajo peso. En cuanto a los aspectos ginecológicos, $62,0 \%$ eran multíparas. Las características socioeconómicas de las madres donantes de leche se pueden ver en la Tabla 1.

Todas las muestras fueron tomadas después de los 30 días de iniciada la lactancia (leche madura), con una mediana de 57 (33-70) días.

La mediana de vitamina A en la leche madura fue de $1,80 \mu \mathrm{mol} / \mathrm{L}(1,36-2,30)$. 
Al analizarse la adecuación a las recomendaciones nutricionales para los lactantes de 0 a 6 meses de edad, considerando las DRI y sabiendo que la ingesta estimada de leche materna durante los primeros seis meses de vida era de $0,78 \mathrm{~L} /$ día, el contenido de vitamina $\mathrm{A}$ en el $50 \%$ de las leches $(n=40)$ no alcanzó a cubrir dichas recomendaciones ( $400 \mu \mathrm{g}$ de retinol/ día).

No se encontró correlación significativa entre el tiempo de lactancia en días y el contenido de vitamina A en la leche (p 0,1407).

\section{DISCUSIÓN}

En este estudio, se determinó la mediana de vitamina A en la leche materna madura. El valor hallado fue de $1,80 \mu \mathrm{mol} / \mathrm{L}(1,36-2,30)$. Solo el $50 \%$ de las leches maduras estudiadas cubrieron las recomendaciones dadas por las DRI para los lactantes de 0 a 6 meses de edad.

Los recién nacidos dependen de la leche materna para cubrir sus necesidades y reservas hepáticas. Hay estudios que indican que las concentraciones de retinol en la leche materna, por lo general, disminuyen a lo largo del primer año posparto. Esto hace que la transferencia de retinol madre-hijo a través de la lactancia

Tabla 1. Características de las madres donantes de leche $(N=79)$

\begin{tabular}{lc}
\hline & N $(\%)$ \\
\hline Ocupación: & $49(62,0)$ \\
Ama de casa & $3(3,8)$ \\
Estudiante & $27(34,2)$ \\
Trabaja fuera de casa & \\
Nivel educativo: & $6(7,6)$ \\
Primario incompleto & $7(8,9)$ \\
Primario completo & $20(25,3)$ \\
Secundario incompleto & $25(31,6)$ \\
Secundario completo & $11(13,9)$ \\
Terc. / univ. incompleto & $10(12,7)$ \\
Terc. / univ. completo & \\
Ingresos familiares: & $4(5,1)$ \\
ARS 10-100 (USD 2-20) & $18(22,8)$ \\
ARS 100-1000 (USD 20-200) & $34(43,0)$ \\
ARS 1000-2000 (USD 200-400) & $23(29,1)$ \\
\hline > ARS 2000 (> USD 400) & $40(50,6)$ \\
\hline Necesidades básicas insatisfechas &
\end{tabular}

ARS: pesos argentinos; USD: dólares estadounidenses. Valor cambiario 2012-2013. materna sea progresivamente menor durante el primer año de vida, ya que la producción media de leche también va disminuyendo entre el primer y duodécimo mes en madres en período de lactancia. Se ha asumido que esta tendencia decreciente de la concentración de retinol en la leche materna, en el posparto, es un signo de agotamiento de las reservas hepáticas maternas, presumiblemente, en relación directa con las concentraciones de vitamina A en la leche materna. ${ }^{9}$ Esto refuerza el concepto de la necesidad de suplir con vitamina A durante la lactancia. ${ }^{10,11}$

La transferencia de vitamina A durante los primeros 6 meses de vida a través de la leche materna es 60 veces mayor que la que ocurre a través de la placenta durante los 9 meses de embarazo. Si el estado nutricional de vitamina A en las madres es deficiente, los niños son susceptibles de sufrir deficiencia de esta vitamina a los 6 meses de edad. Las madres en período de lactancia y los lactantes son considerados población de riesgo para la deficiencia de vitamina A. 10,12,13

Los resultados hallados en nuestro estudio coinciden con dos trabajos publicados por G. Souza y colaboradores, ${ }^{10,14}$ en los que evaluaron el contenido de vitamina A en la leche madura de madres brasileñas asistidas en maternidades públicas, y sus valores hallados fueron 1,76 $\pm 0,85$ y $1,87 \pm 0,81 \mu \mathrm{mol} / \mathrm{L}$, respectivamente. En dichos trabajos, el $50,40 \%$ y el $46,25 \%$ de las leches no cubrían las recomendaciones dadas por las DRI. Sus resultados revelaron una alta prevalencia de un inadecuado estado nutricional de vitamina A de las madres y los niños, en consistencia con la prevalencia nacional reportada en mujeres en edad fértil y los niños brasileños.

Cuando Oliveira y Marinho, evaluaron leche fresca madura de 50 madres donantes de un banco de leche de Manaus, también encontraron que el $58,40 \%$ de ellas no cubrían las recomendaciones dadas para los lactantes. ${ }^{15}$

Tomando en cuenta nuestros resultados, en los que un alto porcentaje de las leches maternas no se adecuaron a las recomendaciones de vitamina $A$, estudiar el estado nutricional de la madre y poder intervenir desde el embarazo mejoraría la calidad de la leche materna y, por consiguiente, el estado nutricional del lactante con lactancia materna exclusiva.

Las limitaciones de nuestro trabajo fueron no haber estudiado el estado nutricional de vitamina A de la madre y el bebé. Además, los resultados 
solo son representativos para leche madura de mujeres asistidas en un hospital público de la ciudad de La Plata.

\section{CONCLUSIÓN}

El estudio demostró que la mitad de las leches de madres estudiadas no aportaban cantidades suficientes de vitamina A para cumplir con las recomendaciones dadas por la Academia Nacional de Ciencias de Estados Unidos.

\section{REFERENCIAS}

1. Sherwin JC, Reacher MH, Dean WH, et al. Epidemiology of vitamin A deficiency and xerophthalmia in at-risk populations. Trans R Soc Trop Med Hyg 2012;106(4):205-14.

2. Kam RK, Deng Y, Chen Y, et al. Retinoic acid synthesis and functions in early embryonic development. Cell Biosci 2012;2(1):11.

3. World Health Organization and Food and Agriculture Organization of the United Nations. Vitamin and mineral requirements in human nutrition. $2^{\text {nd }} \mathrm{ed}$. Hong kong: Joint $\mathrm{FAO} / \mathrm{WHO} ; 2004$.

4. Fernandes TF, Andreto LM, Vieira CS, et al. Serum Retinol Concentrations in Mothers and Newborns at Delivery in a Public Maternity Hospital in Recife, Northeast Brazil. J Health Popul Nutr 2014;32(1):28-35.

5. Engle-Stone R, Haskell MJ, Nankap M, et al. Breast milk retinol and plasma retinol-binding protein concentrations provide similar estimates of vitamin A deficiency prevalence and identify similar risk groups among women in Cameroon but breast milk retinol underestimates the prevalence of deficiency among young children. J Nutr 2014;144(2):209-17.
6. Comisión de Vitaminas de la Sociedad Española de Química Clínica (SEQC). Procedimiento recomendado para la determinación de retinol en suero o plasma. Quím Clin 1998;17(1):38-42.

7. Matamoros N, Santandreu F, Disalvo L, et al. Contenido de vitamina A en leche materna madura después de la pasteurización: Requerimientos nutricionales del lactante. Rev Argent Salud Pública 2014;5(19):11-6.

8. Institute of Medicine. Dietary Reference Intakes: The Essential Guide to Nutrient Requirements. Washington, DC: The National Academies Press; 2006. [Acceso: 20 de septiembre de 2017]. Disponible en: https://doi. org/10.17226/11537.

9. Fujita M, Shell-Duncan B, Ndemwa P, et al. Vitamin A dynamics in breastmilk and liver stores: a life history perspective. Am J Hum Biol 2011;23(5):664-73.

10. SouzaG,DolinskyM,Matos A, etal.Vitamin A concentration in human milk and its relationship with liver reserve formation and compliance with the recommended daily intake of vitamin A in pre-term and term infants in exclusive breastfeeding. Arch Gynecol Obstet 2015;291(2):319-25.

11. KingJC. The risk of maternal nutritional depletion and poor outcomes increases in early or closely spaced pregnancies. J Nutr 2003;133(5 Suppl 2):S1732-6.

12. Stoltzfus RL, Underwood BA. Breast milk vitamin A as an indicator to assess vitamin A status of women and infants. Bull World Health Organ 1995;73(5):703-11.

13. Elmadfa I, Meyer AL. Vitamins for the 1000 days: preparing for life. Int J Vitam Nutr Res 2012;82(5):342-7.

14. Souza G, Saunders C, Dolinsky M, et al. Vitamin A concentration in mature human milk. J Pediatr (Rio J) 2012;88(6):496-502.

15. Oliveira AM, Marinho HA. Determinaçaõ de vitamin A no leite de măes doadoras do banco de leite humano (BLH) de Manaus/AM: efeito do processamento. Acta Amaz 2010;40(1):59-64.

\title{
Anomalías renales en niños con síndrome de Alagille Renal manifestations in children with Alagille syndrome
}

\author{
Dra. Diana Di Pinto y Dra. Marta Adragna ${ }^{a}$
}

a. Servicio de Nefrología, Hospital Nacional de Pediatría "Prof. Dr. Juan P. Garrahan",

Ciudad Autónoma de Buenos Aires, Argentina.

Correspondencia:

Dra. Diana Di Pinto: dianadipinto@yahoo.com

Financiamiento: Ninguno.

Conflicto de intereses: Ninguno que declarar.

Recibido: 13-7-2017

Aceptado: 14-9-2017

\author{
RESUMEN \\ Introducción. El síndrome de Alagille (SA) es una enfermedad \\ colestásica debida a la escasez de los conductos biliares \\ interlobulares. Se asocia con manifestaciones extrahepáticas, \\ y el compromiso renal es frecuente. \\ Objetivos. Describir la prevalencia, tipo y evolución de la \\ patología renal en niños con SA. \\ Pacientes y métodos. Se estudió retrospectivamentela presencia \\ y evolución de la patología renal en 21 niños que reunían \\ criterios de SA. \\ Resultados. En 18 pacientes $(85,7 \%$ ), se observó patología renal: \\ (1) alteraciones ecográficas en 7 (displasia renal bilateral en 6 \\ y agenesia renal en 1); (2) acidosis tubular renal distal en 2; \\ (3) caída de filtrado glomerular y/o proteinuria en 16. La \\ frecuencia de caída de filtrado glomerular fue similar entre \\ pacientes con y sin ecografía renal patológica.
}

